\title{
Synthesis, Characterization and Antimicrobial Activities of 1,2,4-Triazole /lsatin Schiff bases and their $\mathrm{Mn}(\mathrm{II}), \mathrm{Co}(\mathrm{II})$ Complexes
}

\author{
SUNITA BAJROLIYA, G. S. KALWANIA*, SAVITA CHOUDHARY and S. CHOMAL \\ Department of Chemistry, S. K. Government P. G. College Sikar - 332001, India \\ ${ }^{*}$ Corresponding author E-mail: gskalwania@gmail.com
}

http://dx.doi.org/10.13005/ojc/300419

(Received: September 16, 2014; Accepted: October 26, 2014)

\begin{abstract}
$\mathrm{Mn}$ (II) and Co(II) metal complexes have been synthesized with newly synthesized Schiff bases derived from isatin/5-nitroisatin and 3-substituted-4-amino-5-mercapto-1,2,4-triazole by new environmental benign microwave irradiation method as well as conventional method. Reaction achieved by microwave irradiation technique, require drastically reduced reaction time and provide high yield with improved selectivity as compared to conventional method. The synthesized compounds were characterized by elemental analysis as well as spectral studies. The elemental analysis were clearly indicated that $\mathrm{ML}_{2}$ type complexes have 1:2 stoichiometry ( $\mathrm{M}=$ metal, $\mathrm{L}=$ ligand). The synthesized Schiff bases and their metal complexes screened for antimicrobial activities against selected bacteria and fungi.
\end{abstract}

Keywords: Schiff bases, 1,2,4-Triazole, Isatin, 5-Nitroisatin, Microwave irradiation, Antimicrobial, Complexes.

\section{INTRODUCTION}

The chemistry of 1,2,4-triazole and its derivatives is particularly interesting because of their potential application in medicinal, agricultural and industrial fields ${ }^{1-4}$. Derivatives of 1,2,4-triazoles show variety of biological activities such as antimicrobial ${ }^{5}$ anticonvulsant ${ }^{6}$, anticancer ${ }^{7}$, analgesic ${ }^{8}$, anti $\mathrm{HIV}^{9}$, and anti-inflammatory ${ }^{10}$ properties. Similarly, Isatin and Schiff bases of isatin derivatives are reported to show diverse biological activities like antibacterial ${ }^{11}$, antifungal ${ }^{12}$, anticonvulsant ${ }^{13}$, anti-HIV ${ }^{14}$, antidepressant ${ }^{15}$ and anti-inflammatory ${ }^{16}$ activities. It is well known that the heterocyclic compounds containing both 1,2,4-triazole and isatin rings have broad spectrum of pharmacologic properties ${ }^{2-8,11-15 \text {, }}$ 17-18. Some metal complexes derived from isatin and 1,2,4-triazole Schiff bases have reported to exhibit antimicrobial activities ${ }^{19}$. These diverse biological applications of schiff bases and their precursors have inspired us to synthesize a new series of [Mn(II), Co(II)] metal complexes with Schiff bases containing isatin/5-nitroisatin and 3-substituted-4amino-5-mercapto-1,2,4-triazole. 
In recent years Microwave assisted reactions have attracted researchers much interest because of simplicity of operation, enhanced reaction rates, high yields, improved selectivity $20-21$ and environmental friendly reaction condition ${ }^{22-24}$. The reactions, which are not possible under normal conditions, can be overcome by high-energy microwave irradiation. Thereforwe have made efforts to develop and optimize anew environmentally benign method using microwave irradiation in our laboratory and finally 3 -substituted4-amino-5-mercapto-1,2,4-triazole ${ }^{25}$ and isatin derivatives have been synthesized. These compounds were also prepared by conventional methods ${ }^{26}$ to compare their results with new microwave irradiation method. The newly synthesized compounds were characterized by spectral as well as elemental analysis and screened for their biological activities.

\section{EXPERIMENTAL}

The melting point of synthesized compounds were determined on open aluminum block and not corrected. Purity was checked by TLC (thin layer of silica gel) using Merck Silica gel G-60. Infrared (IR) spectra of all the synthesized compounds recorded using $\mathrm{KBr}$ from Shimadzu FTIR, Affinity-1. The NMR spectra has taken by Varian Gemini 400 spectrometer $(300 \mathrm{MHz})$ using TMS as an internal standard. Elemental data obtained from all the synthesized compounds were found satisfactory.

\section{RESULTS AND DISCUSSION}

The focus of the present work was on the synthesis of some new Schiff bases and their Mn (II), Co (II) metal complexes by microwave irradiation (Scheme 1). These compounds were also synthesized by conventional method ${ }^{26}$. In microwave method, the reaction time reduced from 6 hrs.to 3-5 minutes (3a-h) and from 4 hrs.to 4-7 minutes (4a-p). The yield obtained from microwave method was better as compared to conventional method. The purity of the compounds was checked by TLC. The compounds were characterized with the help of spectral data (IR, ${ }^{1} \mathrm{H}$ NMR) and elemental analysis. All data were found in accordance with the proposed structures of synthesized compounds. The IR spectroscopic data have indicated the formation of 3a-h by disappearance of the $\mathrm{NH}_{2}$ band at 3400 $\mathrm{cm}^{-1}$ (2a-d) and appearance of $\mathrm{C}=\mathrm{N}$ band at about $1608-1643 \mathrm{~cm}^{-1}$, The stretching vibrational $\mathrm{N}-\mathrm{H}$ band at about $3201-3271 \mathrm{~cm}^{-1}$ and $\mathrm{C}=\mathrm{O}$ band at about $1707-1738 \mathrm{~cm}^{-1}$ confirm the presence of indole ring in compounds $3 \mathrm{a}-\mathrm{h}$. A characteristic strong band at $2690-2716 \mathrm{~cm}^{-1}$ clearly indicate the presence of $\mathrm{S}-\mathrm{H}$ group intriazole and another band around at $1098-1112 \mathrm{~cm}^{-1}$ is assigned to $v(\mathrm{C}=\mathrm{S})^{27}$. These observations indicate that the Schiff bases exhibit thiol-thionetautomerism.

All the metal complexes exhibited the band of $v(\mathrm{C}=\mathrm{N})$ in the region $1592-1630 \mathrm{~cm}^{-1}$, showing the shift of this band to lower wave numbers, as compared with the spectra of the Schiff bases. This shift indicates that the nitrogen is coordinated to the metal ion ${ }^{28-30}$. The band of $v(\mathrm{C}=\mathrm{O})$ in the region $1702-1730 \mathrm{~cm}^{\prime 1}$ in the complexes, showing the shift to lower wave numbers compared with Schiff bases confirms that the carbonyl oxygen is also coordinated to the metal ion ${ }^{31-32}$. The new bands in the region of $460-472 \mathrm{~cm}^{-1}$ in the spectra of the metal complexes are assigned to stretching frequencies of $(\mathrm{M}-\mathrm{N})$ bonds. The absence of a band around at $2700 \mathrm{~cm}^{\text {"11 }}$ in all the metal complexes and new band in the region $358-380 \mathrm{~cm}^{\text {"1 }}$ in far IRspectra indicate the formation of metal-sulfur bonds. All the infrared frequencies of metal complexes are summarized in Table 2.The comparison of ${ }^{1} \mathrm{H}$ NMR spectroscopic data of Schiff bases and their precursors also indicate the formation of $3 a-h$ by the appearance of signal corresponding of two different $\mathrm{NH}$ protons at about $11.10-12.50 \mathrm{ppm}$ and the disappearance of the $\mathrm{NH}_{2}$ protons in the $2 \mathrm{a}-\mathrm{d}$ at about $5.00 \mathrm{ppm}$.

All the Schiff bases (3a-h) and their metal complexes (4a-p)along with some standard antibiotics and antifungal drugs were screened for their antimicrobial activities against selected bacteria and fungus. All the antimicrobial activity results are summarized in Table 3 . The nitro derivatives of Schiff bases (3e-h) were found more active as compared to others. Co (II) complexes (4ip) show higher antimicrobial activity in comparison to respective Schiff bases against selected bacteria and fungi. 


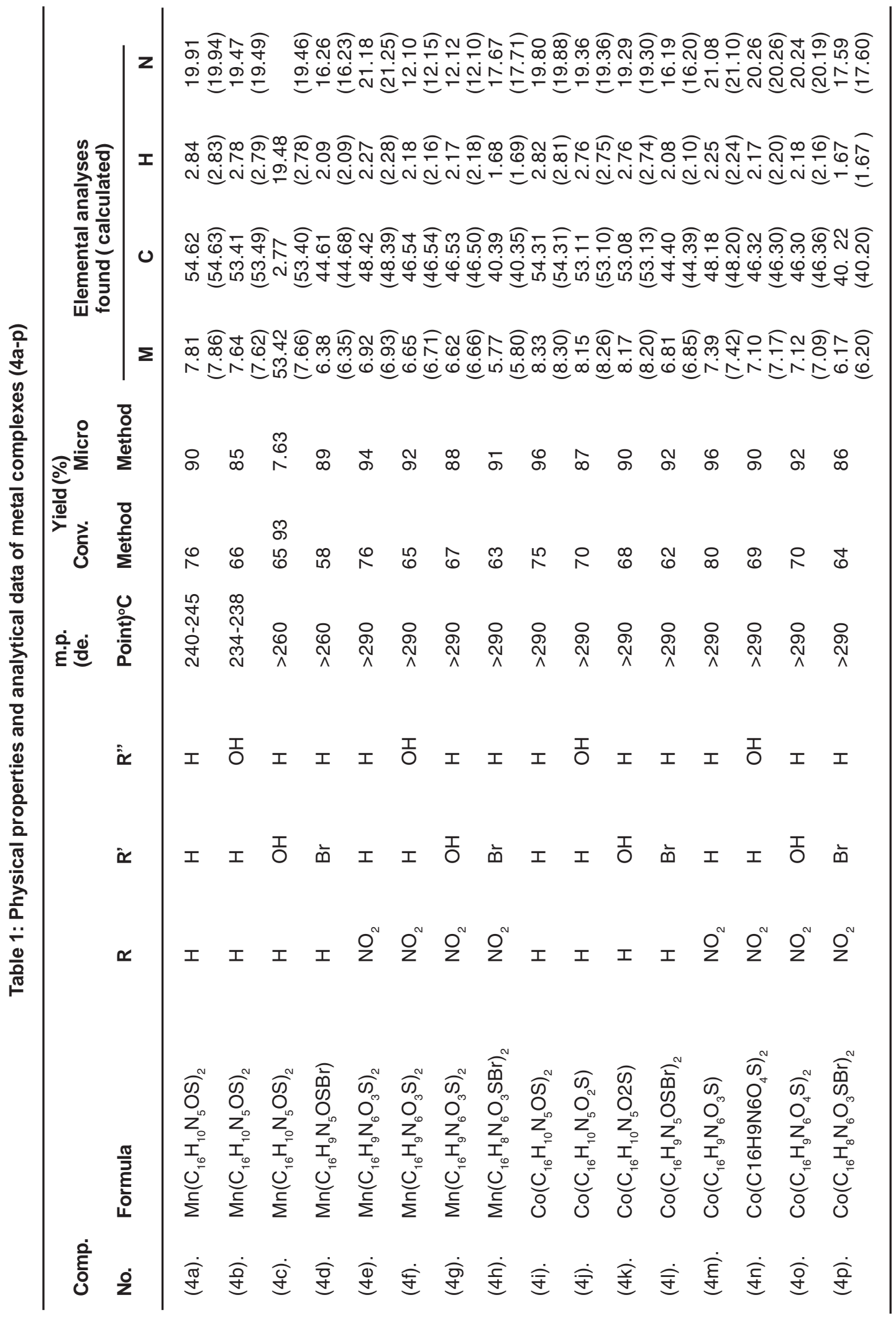


General Method for the Synthesis of Schiff bases All the Schiff bases were synthesized by both conventional and microwave assisted methods. The latter method provided reproducible results and analytical data related to the products obtained from both methods were found coherent.

\section{Conventional Method}

The equimolar quantity of 3-substituted4-amino-5-mercapto-1,2,4-triazole (10 mmol) and isatin/5-nitroisatin (10 mmol) containing 3-4 drops concentrated $\mathrm{HCl}$ in hot methanol $(40 \mathrm{ml})$ was refluxed for 6 hrs. After completion of reaction, the reaction mixture was cooled at room temperature. This solid obtained was filtered, washed with cold methanol, dried and recrystallized from methanol.

\section{Microwave Assisted Method}

A mixture of 3-substituted-4-amino-5mercapto-1,2,4-triazole $(1 \mathrm{~m} \mathrm{~mol})$ and isatin/5nitroisatin $(1 \mathrm{mmol})$ in methanol $(10 \mathrm{ml})$ containing concentrated $\mathrm{HCl}$ (3-4 drops) was subjected to microwave irradiation intermittently at 30 seconds for 3-5 minutes. After completion of reaction as monitored by TLC, the reaction mixture was cooled at room temperature. The solid separated out on cooling was filtered, washed with cold methanol, dried and recrystallized from methanol.

The analytical data for all the synthesizes Schiff bases are summarized here. The yield obtained by microwave -assisted method is represented in bracket.

\section{3-[3-Phenyl-2',4'-dihydro-1',2',4'-triazol-5'-} mercapto-4'-yl]iminoisatin

Yield $72 \%$, (95\%); m.p. $221^{\circ} \mathrm{C}$; IR ( $\mathrm{KBr}$, $\left.v_{\text {max. }}, \mathrm{cm}^{-1}\right): 1728(>\mathrm{C}=\mathrm{O}), 1614(\mathrm{C}=\mathrm{N}), 3211(\mathrm{~N}-\mathrm{H}$, isatin), $3187(\mathrm{~N}-\mathrm{H}$, triazole), $2715(\mathrm{~S}-\mathrm{H}), 1104$ $(\mathrm{C}=\mathrm{S})$; ${ }^{1} \mathrm{H}-\mathrm{NMR}$ (DMSO-d $\left.{ }_{6},{ }^{\prime} \mathrm{ppm}\right)$ : 6.96-7.80 (Ar$9 \mathrm{H}, \mathrm{m}$ ), 11.98 (isatin, $\mathrm{N}-\mathrm{H}, 1 \mathrm{H}, \mathrm{s}), 11.14$ (triazole, $\mathrm{N}-\mathrm{H}, 1 \mathrm{H}, \mathrm{s}), 11.20(\mathrm{~S}-\mathrm{H}, 1 \mathrm{H}, \mathrm{s})$; Anal. Calculated (\%) for $\mathrm{C}_{16} \mathrm{H}_{11} \mathrm{~N}_{5} \mathrm{OS}$; C: 59.82, $\mathrm{H}: 3.41, \mathrm{~N}: 21.80$; Found (\%); C: 59.81, H: 3.40, N: 21.80.

3-[3'-(2''-Hydroxyphenyl)-2',4'-dihydro-1',2',4'triazol-5'-mercapto-4'-yl]iminoisatin

Yield $64 \%$, (91\%); m.p. $178^{\circ} \mathrm{C}$; IR $(\mathrm{KBr}$, $\left.v_{\text {max. }}, \mathrm{cm}^{-1}\right): 1730(>\mathrm{C}=\mathrm{O}), 1613(\mathrm{C}=\mathrm{N}), 3208(\mathrm{~N}-\mathrm{H}$, isatin), $3165(\mathrm{~N}-\mathrm{H}$, triazole), $2706(\mathrm{~S}-\mathrm{H}), 1106$
(C=S), 756 (1,2-disubstitutedphenyl); ${ }^{1} \mathrm{H}-\mathrm{NMR}$ (DMSO-d ${ }_{6}$ ' ppm): 6.93-7.78 (Ar-8H, m), 11.92 (isatin, $\mathrm{N}-\mathrm{H}, 1 \mathrm{H}, \mathrm{s}$ ), 11.12 (triazole, $\mathrm{N}-\mathrm{H}, 1 \mathrm{H}, \mathrm{s}$ ), 11.18 (S-H, $1 \mathrm{H}, \mathrm{s})$; Anal. Calculated (\%) for $\mathrm{C}_{16} \mathrm{H}_{11} \mathrm{~N}_{5} \mathrm{O}_{2} \mathrm{~S}$; C: 56.97, H: 3.26, N: 20.78; Found (\%); C: 56.97, H: 3.26, N: 20.77 .

3-[3'-(4'--Hydroxyphenyl)-2',4'-dihydro-1',2',4'triazol-5'-mercapto-4'-yl]iminoisatin

Yield 66\%, (92\%); m.p. $249{ }^{\circ} \mathrm{C}$; IR $(\mathrm{KBr}$, $\left.v_{\text {max. }}, \mathrm{cm}^{-1}\right): 1731(>\mathrm{C}=\mathrm{O}), 1614(\mathrm{C}=\mathrm{N}), 3209(\mathrm{~N}-\mathrm{H}$, isatin), $3168(\mathrm{~N}-\mathrm{H}$, triazole), $2705(\mathrm{~S}-\mathrm{H}), 1105$ $(\mathrm{C}=\mathrm{S}), 841$ (1,4-disubstitutedphenyl); ${ }^{1} \mathrm{H}-\mathrm{NMR}$ (DMSO-d ${ }_{6}, '$ ppm): 7.95-7.80 (Ar-8H, m), 11.91
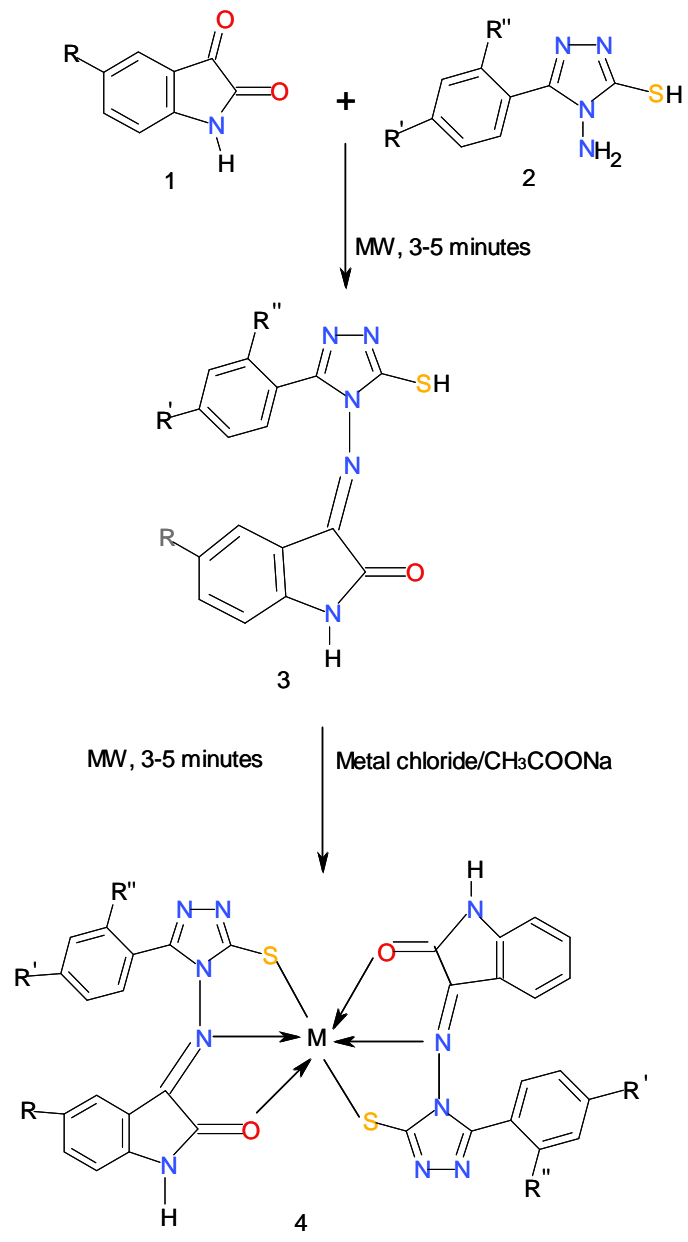

$\mathrm{R}=\mathrm{H}, \mathrm{NO}_{2} ; \mathrm{R}^{\prime}=\mathrm{H}, \mathrm{OH}, \mathrm{Br} ; \mathrm{R}=\mathrm{H}, \mathrm{OH} ; \mathrm{M}=\mathrm{Mn}(\mathrm{II}), \mathrm{Co}(\mathrm{II})$

Scheme 1: Synthesis pathway for the preparation of compounds 
(isatin, $\mathrm{N}-\mathrm{H}, 1 \mathrm{H}, \mathrm{s}$ ), 11.13 (triazole, $\mathrm{N}-\mathrm{H}, 1 \mathrm{H}, \mathrm{s}$ ), 10.85 $(\mathrm{S}-\mathrm{H}, 1 \mathrm{H}, \mathrm{s})$; Anal. Calculated (\%) for $\mathrm{C}_{16} \mathrm{H}_{11} \mathrm{~N}_{5} \mathrm{O}_{2} \mathrm{~S}$; C: $56.97, \mathrm{H}: 3.27, \mathrm{~N}: 20.77$; Found (\%); C: 56.97, H : $3.26, \mathrm{~N}: 20.79$.

3-[3'-(4''-Bromophenyl)-2',4'-dihydro-1',2',4'triazol-5'-mercapto-4'-yl]iminoisatin

Yield 62\%, (88\%); m.p. $260^{\circ} \mathrm{C}$; IR $(\mathrm{KBr}$, $\left.v_{\text {max. }}, \mathrm{cm}^{-1}\right): 1726(>\mathrm{C}=\mathrm{O}), 1608(\mathrm{C}=\mathrm{N}), 3201(\mathrm{~N}-\mathrm{H}$, isatin), $3134(\mathrm{~N}-\mathrm{H}$, triazole), $2690(\mathrm{~S}-\mathrm{H}), 1098$ $(\mathrm{C}=\mathrm{S}), 845$ (1,4-disubstitutedphenyl); ${ }^{1} \mathrm{H}-\mathrm{NMR}$ (DMSO-d $\mathrm{d}_{6}$ ' ppm): 7.08-7.90 (Ar-8H, m), 12.00 (isatin, $\mathrm{N}-\mathrm{H}, 1 \mathrm{H}, \mathrm{s}$ ), 11.15 (triazole, $\mathrm{N}-\mathrm{H}, 1 \mathrm{H}, \mathrm{s}$ ), 10.98 (S-H, $1 \mathrm{H}, \mathrm{s}$ ); Anal. Calculated (\%) for $\mathrm{C}_{16} \mathrm{H}_{10} \mathrm{~N}_{5} \mathrm{OSBr}$ ; C: 48.03, H: 2.50, N: 17.52; Found (\%); C: 48.01, H :2.50, N: 17.50 .

3-[3'-Phenyl-2',4'-dihydro-1',2',4'-triazol-5'mercapto-4'-yl]imino-5-nitroisatin

Yield $70 \%$, (90\%); m.p. $269^{\circ} \mathrm{C}$; IR $(\mathrm{KBr}$ $\left.v_{\max }, \mathrm{cm}^{-1}\right): 1707(>\mathrm{C}=\mathrm{O}), 1632(\mathrm{C}=\mathrm{N}), 3265(\mathrm{~N}-\mathrm{H}$, isatin), $3121(\mathrm{~N}-\mathrm{H}$, triazole), $2716(\mathrm{~S}-\mathrm{H}), 1106$ $(\mathrm{C}=\mathrm{S}), 13551541\left(\mathrm{NO}_{2}\right)$; ${ }^{1} \mathrm{H}-\mathrm{NMR}$ (DMSO-d ${ }_{6}$, ppm): 7.12-8.40 (Ar-8H, m), 12.42 (isatin, $\mathrm{N}-\mathrm{H}, 1 \mathrm{H}$, s), 11.81 (triazole, $\mathrm{N}-\mathrm{H}, 1 \mathrm{H}, \mathrm{s}), 10.97(\mathrm{~S}-\mathrm{H}, 1 \mathrm{H}, \mathrm{s})$; Anal. Calculated (\%) for $\mathrm{C}_{16} \mathrm{H}_{10} \mathrm{~N}_{6} \mathrm{O}_{3} \mathrm{~S}$; C: $52.44, \mathrm{H}$ : 2.73, N: 22.96; Found (\%); C: 52.45, H: 2.73, N: 22.95.
3-[3'-(2'-Hydroxyphenyl)-2',4'-dihydro-1',2',4' triazol-5'-mercapto-4'yl]imino-5-nitroisatin

Yield $65 \%$, (86\%); m.p. $290^{\circ} \mathrm{C}$; IR $(\mathrm{KBr}$, $\left.v_{\max }, \mathrm{cm}^{-1}\right): 1714(>\mathrm{C}=\mathrm{O}), 1641(\mathrm{C}=\mathrm{N}), 3271(\mathrm{~N}-\mathrm{H}$, isatin), $3116(\mathrm{~N}-\mathrm{H}$, triazole $), 2710(\mathrm{~S}-\mathrm{H}), 1110$ $(\mathrm{C}=\mathrm{S}), 764$ (1,2-disubstitutedphenyl), 13501542 $\left(\mathrm{NO}_{2}\right)$; ${ }^{1} \mathrm{H}-\mathrm{NMR}$ (DMSO-d $\left.\mathrm{d}_{6},{ }^{\mathrm{p} p m}\right)$ : 7.39-8.16 (Ar$7 \mathrm{H}, \mathrm{m}$ ), 12.29 (isatin, $\mathrm{N}-\mathrm{H}, 1 \mathrm{H}, \mathrm{s}), 11.62$ (triazole, $\mathrm{N}-\mathrm{H}, 1 \mathrm{H}, \mathrm{s}), 11.20(\mathrm{~S}-\mathrm{H}, 1 \mathrm{H}, \mathrm{s})$; Anal. Calculated (\%) for $\mathrm{C}_{16} \mathrm{H}_{10} \mathrm{~N}_{6} \mathrm{O}_{4} \mathrm{~S}$; C: $50.27, \mathrm{H}: 2.61, \mathrm{~N}: 21.97$; Found (\%); C: 50.26, H : 2.61, N: 21.98.

3-[3'-(4''-Hydroxyphenyl)-2',4'-dihydro-1',2',4'triazol-5'-mercapto-4'-yl]imino-5-nitroisatin

Yield $67 \%$, (94\%); m.p. $280^{\circ} \mathrm{C}$; IR $(\mathrm{KBr}$, $\left.v_{\max .}, \mathrm{cm}^{-1}\right): 1718(>\mathrm{C}=\mathrm{O}), 1640(\mathrm{C}=\mathrm{N}), 3268(\mathrm{~N}-\mathrm{H}$, isatin), $3168(\mathrm{~N}-\mathrm{H}$, triazole), $2708(\mathrm{~S}-\mathrm{H}), 1110(\mathrm{C}=\mathrm{S})$, 844 (1,4-disubstitutedphenyl), $13501542\left(\mathrm{NO}_{2}\right) ; \mathrm{H}-$ NMR (DMSO-d , ' ppm): 7.35-8.18 (Ar-7H, m), 12.30 (isatin, $\mathrm{N}-\mathrm{H}, 1 \mathrm{H}, \mathrm{s}), 11.59$ (triazole, $\mathrm{N}-\mathrm{H}, 1 \mathrm{H}, \mathrm{s}), 10.96$ (S-H, $1 \mathrm{H}, \mathrm{s})$; Anal. Calculated (\%) for $\mathrm{C}_{16} \mathrm{H}_{10} \mathrm{~N}_{6} \mathrm{O}_{4} \mathrm{~S}$; C: 50.27, H: 21.98, N: 20.77; Found (\%); C: 50.27, H :2.60, N: 21.99 .

3-[3'-(4''-Bromophenyl)-2',4'-dihydro-1',2',4'triazol-5'-mercapto-4'-yl]imino-5-nitroisatin

Yield $60 \%$, (90\%); m.p. $292^{\circ} \mathrm{C}$; IR $(\mathrm{KBr}$,

Table 2: Characteristic infrared vibrations $\left(\mathrm{cm}^{-1}\right)$ of metal complexes (4a-p)

\begin{tabular}{lccccc}
\hline Compound & \multicolumn{5}{c}{ Indole ring } \\
\cline { 2 - 6 } No. & $v(\mathrm{C}=\mathrm{O})$ & $v(\mathrm{C}=\mathrm{N})$ & $v(\mathrm{~N}-\mathrm{H})$ & $v(\mathrm{M}-\mathrm{S})$ & $v(\mathrm{M}-\mathrm{N})$ \\
\hline & 1718 & 1601 & 3210 & 364 & 465 \\
$4(\mathrm{a})$ & 1721 & 1599 & 3206 & 359 & 468 \\
$4(\mathrm{~b})$ & 1716 & 1596 & 3210 & 361 & 466 \\
$4(\mathrm{~d})$ & 1711 & 1593 & 3203 & 376 & 463 \\
$4(\mathrm{e})$ & 1704 & 1612 & 3217 & 365 & 464 \\
$4(\mathrm{f})$ & 1705 & 1627 & 3198 & 358 & 470 \\
$4(\mathrm{~g})$ & 1703 & 1622 & 3211 & 363 & 464 \\
$4(\mathrm{~h})$ & 1727 & 1630 & 3197 & 374 & 462 \\
$4(\mathrm{i})$ & 1720 & 1603 & 3213 & 367 & 464 \\
$4(\mathrm{j})$ & 1722 & 1596 & 3207 & 358 & 466 \\
$4(\mathrm{k})$ & 1719 & 1598 & 3211 & 363 & 467 \\
$4(\mathrm{l})$ & 1709 & 1592 & 3198 & 379 & 461 \\
$4(\mathrm{~m})$ & 1702 & 1617 & 3216 & 371 & 465 \\
$4(\mathrm{n})$ & 1709 & 1628 & 3201 & 365 & 463 \\
$4(\mathrm{o})$ & 1707 & 1620 & 3210 & 360 & 468 \\
$4(\mathrm{p})$ & 1730 & 1628 & 3202 & 378 & 460 \\
\hline
\end{tabular}


$\left.v_{\text {max. }}, \mathrm{cm}^{-1}\right): 1738(>\mathrm{C}=\mathrm{O}), 1643(\mathrm{C}=\mathrm{N}), 3230(\mathrm{~N}-\mathrm{H}$, isatin), $3130(\mathrm{~N}-\mathrm{H}$, triazole), $2702(\mathrm{~S}-\mathrm{H}), 1112$ $(\mathrm{C}=\mathrm{S}), 852$ (1,4-disubstitutedphenyl), 13621540 $\left(\mathrm{NO}_{2}\right)$; ${ }^{1} \mathrm{H}-\mathrm{NMR}$ (DMSO-d ${ }_{6}$, ' ppm): 7.23-8.65 (Ar$7 \mathrm{H}, \mathrm{m}$ ), 12.49 (isatin, $\mathrm{N}-\mathrm{H}, 1 \mathrm{H}, \mathrm{s}), 11.96$ (triazole, $\mathrm{N}$ $\mathrm{H}, 1 \mathrm{H}, \mathrm{s}), 10.90$ (S-H, 1H, s); Anal. Calculated (\%) for $\mathrm{C}_{16} \mathrm{H}_{9} \mathrm{~N}_{6} \mathrm{O}_{3} \mathrm{SBr}$; C: 43.15, $\mathrm{H}: 2.04, \mathrm{~N}: 18.87$; Found (\%); C: 43.15, H:2.02, N: 18.88.

\section{General Method for the Synthesis of Metal Complexes}

All the metal complexes were synthesized by both conventional and microwave assisted methods. The latter method provided reproducible results and analytical data related to the products obtained from both methods were found coherent.

\section{Conventional method}

A solution of metal chloride $(1 \mathrm{mmol})$ in methanol $(6 \mathrm{ml})$ and $2 \mathrm{mmol}$ of sodium acetate were added to Schiff base ( $2 \mathrm{mmol})$ dissolved in Methanol $(30 \mathrm{ml})$ and refluxed on water bath for $4 \mathrm{hrs}$. The reaction mixture was cooled at room temperature and solid separated out was filtered, washed thoroughly with methanol and water and dried.

\section{Microwave Assisted Method}

A solution of metal chloride $(1 \mathrm{mmol})$ in methanol $(3 \mathrm{ml})$ and $2 \mathrm{mmol}$ of sodium acetate were added to Schiff base ( $2 \mathrm{mmol}$ ) dissolved in Methanol $(10 \mathrm{ml})$ and subjected to microwave irradiation intermittently at 30 seconds for 4-7 minutes. After completion of the reaction as monitored by TLC, the

Table 3: Antimicrobial activities of schiff bases and their metal complexes

\begin{tabular}{|c|c|c|c|c|c|c|}
\hline \multirow{3}{*}{ Compd.No. } & \multicolumn{5}{|c|}{ Zone of Inhibition in $\mathrm{mm}$} & \\
\hline & \multicolumn{3}{|c|}{ Antibacterial Activity } & \multicolumn{3}{|c|}{ Antifungal Activity } \\
\hline & E. coli & P. aeruginosa & S. aureus & B. subtilis & A. niger & C. albicans \\
\hline $3(a))$ & 7 & 8 & 9 & 9 & 8 & 7 \\
\hline $3(b)$ & 6 & 7 & 8 & 7 & 7 & 7 \\
\hline 3(c) & 7 & 9 & 7 & 10 & 6 & 8 \\
\hline $3(d)$ & 8 & 9 & 7 & 6 & 9 & 7 \\
\hline $3(e)$ & 7 & 11 & 10 & 13 & 8 & 8 \\
\hline $3(f)$ & 8 & 10 & 11 & 15 & 10 & 9 \\
\hline $3(\mathrm{~g})$ & 8 & 12 & 10 & 10 & 8 & 9 \\
\hline $3(\mathrm{~h})$ & 10 & 9 & 12 & 13 & 11 & 10 \\
\hline $4(a)$ & 7 & 10 & 6 & 7 & 8 & 10 \\
\hline $4(b)$ & 8 & 8 & 10 & 9 & 6 & 7 \\
\hline $4(c)$ & 9 & 8 & 9 & 9 & 8 & 6 \\
\hline 4(d) & 7 & 9 & 7 & 8 & 6 & 6 \\
\hline $4(e)$ & 8 & 7 & 6 & 10 & 8 & 8 \\
\hline $4(\mathrm{f})$ & 6 & 11 & 8 & 8 & 8 & 9 \\
\hline $4(\mathrm{~g})$ & 7 & 8 & 9 & 9 & 7 & 6 \\
\hline $4(h)$ & 8 & 10 & 12 & 6 & 10 & 9 \\
\hline $4(\mathrm{i})$ & 8 & 10 & 8 & 9 & 7 & 7 \\
\hline $4(j)$ & 9 & 9 & 7 & 9 & 8 & 11 \\
\hline $4(k)$ & 8 & 10 & 7 & 12 & 7 & 9 \\
\hline $4(I)$ & 7 & 8 & 8 & 9 & 8 & 8 \\
\hline $4(m)$ & 9 & 13 & 11 & 14 & 10 & 9 \\
\hline $4(n)$ & 11 & 10 & 13 & 16 & 10 & 11 \\
\hline $4(0)$ & 10 & 13 & 14 & 12 & 9 & 9 \\
\hline $4(p)$ & 12 & 11 & 16 & 15 & 9 & 10 \\
\hline Streptomycein & 18 & 20 & 23 & 25 & - & - \\
\hline Fluconazole & - & - & - & - & 23 & 22 \\
\hline
\end{tabular}


hot reaction mixture was cooled at room temperature. The solid separated out was filtered, washed thoroughly with methanol and water and dried.

\section{Antimicrobial activity}

Antimicrobial activity of synthesized Schiff bases and their metal complexes were evaluated in vitro by using agar-plate diffusion technique ${ }^{33}$ by measuring the zone of inhibition in $\mathrm{mm}$. The antibacterial activity was evaluated against four bacterial strains Escherichia coli ATCC 25922(E. coli), Pseudomonas aeruginosa ATCC 27853 ( $P$. aeruginosa), Staphylococcus aureus ATCC 25923 (s. aureus) and Bacillus subtilis ATCC 6633 (B. subtilis) at $1001 / \mathrm{g} / \mathrm{mL}$ concentration of samples with standard drugs Streptomycin. After completion of incubation period $\left(18-24 \mathrm{~h}\right.$ at $\left.37^{\circ} \mathrm{C}\right)$, the zone of inhibition of growth in the form of diameter in $\mathrm{mm}$ was measured (Table 3).

An antifungal activity was evaluated against Aspergillus neiger (A. neiger) and Canadida albicans (C. albicans) at $100 \mu \mathrm{g} / \mathrm{mL}$ concentration of samples using Fluconazole as standard drug. After completion of the incubation period (18-24 $\mathrm{h}$ at $37^{\circ} \mathrm{C}$ ), the zone of inhibition of growth in the form of diameter in $\mathrm{mm}$ was measured (Table 3 ).

\section{CONCLUSION}

The prepared Schiff bases behave as tridentate chelating agent with $\mathrm{Mn}(\mathrm{II}), \mathrm{Co}(\mathrm{II})$ metal ions and coordinated through $\mathrm{N}, \mathrm{O}$ and $\mathrm{S}$ atoms. The elemental analysis were clearly indicated that $\mathrm{ML}_{2}$ type complexes have 1:2 stoichiometry ( $M=$ metal, $\mathrm{L}=$ ligand). All the Schiff bases and their $\mathrm{Mn}(\mathrm{II}), \mathrm{Co}(\mathrm{II})$ metal complexes were synthesized by microwave irradiation as well as by conventional method to compare analytical data and confirm their structure. The studies revealed that Schiff bases containing nitro group were found more active against selected bacteria and fungi. It is also observed that Co (II) metal complexes have exhibited higher antimicrobial activity than the respective Schiff bases as cleared from data in table 3.

\section{ACKNOWLEDGEMENT}

We are thankful to the Principal of Institute and head of the chemistry department for providing necessary facilities to complete this study. One of the authors,Sunita Kumari Bajroliyais also thankful to UGC for financial support in the form of JRF.

\section{REFERENCES}

1. Legrand, C.; Ropaa, P.; Vulturescu, B.; Hogeac, A. M.; Surpateanu, G.G.; Cazier, F.; Woisel, P.; Surpateanu, G. Progress in Organic Coatings 2005, 53, 106-111.

2. Ewiss, N. F.; Bahajaj, A. A.; Elsherbini, E. A. J. Heterocycl. Chem.1986, 23, 1451-1458.

3. Eweiss, N. F.; Bahajaj, A. A. J. Heterocycl. Chem.1987, 24, 1173-1182.

4. Prasad, A.; Ramalingam, R. J.; Rao, A. B.; Diwan, P. V.; Sattur, P. B. Eur. J. Med.Chem. 1989, 24, 199-201.

5. Turan-Zitouni, G.; Kaplanc1kl1, Z. A.;Y1ld1z, M. T.; Chevallet, P.; Kaya, D. Eur. J. Med.Chem.2005, 40, 607-613.

6. Chen, J.; Sun, Y.; Chai, K. Y.; Lee, J. S.; Song, M. S.; Quan, Z. S. Bioorg. Med. Chem. 2007,15, 6775-6781.

7. Bekircan, O.; Gumrukcuoglu, N. Indian J. Chem. 2005, 44B, 2107-2113.
8. Turan-Zitouni, G.; Kaplanc1kl1, Z. A.; Erol, K.; Kilic, F. S. Farmaco1999, 54, 218-223.

9. Akhtar, T.; Hameed, S.; Al-Masoudi, N. A.; Khan, K. M. Heteroatom. Chem. 2007, 18, 316-322.

10. Turan-Zitouni, G.; Kaplancikli, Z. A.; Özdemir, A.; Chevallet, P. Arch. Pharm. Chem. Life Sci .2007,340,586-590.

11. Sarangapani, M.; Reddy, V. M.Indian J. Pharm. Sci.1994,56, 174-177.

12. Pandeya, S. N.; Sriram, D.; Nath, G.; De Clercq, E. Pharm. ActaHelv.1999, 74, 11-17

13. Popp, F. D.; Parson, R.; Donigan, B. E. J. Heterocycl. Chem. 1980, 17, 1329-1330.

14. Pandeya, S. N.; Sriram D.; DeClercq, E.; Nath, G. Eur. J. Pharm. Sci.1999,9, 25-31.

15. Singh, G. S.; Singh, T.; Lakhan, R. Indian J. Chem. 1997, 36B, 951-954. 
16. Bhattacharya, S. K.; Chakrabarti, S. Indian J. Exp. Biol. 1998, 36, 118-121.

17. Islam, M. R.; Abedin, M. J.; Hossain, M. M.; Duddeck H. J. Bangladesh Chem. Soc.1998,11,71-78

18. Kahveci, B. Molecules2005, 10, 376-382.

19. Kulkarni, D.; Patil, S. A.; Badami, P. S. Journal of Sulfur Chemistry 2009,30(2), 145-159.

20. Chandak, S.Tetrahedron1995, 51, 1040310432.

21. Kidwa, M.; Sapra, P.; Bhushan, K. R.; Saxena, R. K.Montash Chem. 2000, 113, 85-90.

22. Kappe, C. O.Curr.Opin.Chem. Biol.2002, 6, 314-320.

23. Larhed, M.; Moberg, C.; Hallberg, A.Acc. Chem. Res. 2002,35, 717-727.

24. Lidstrom, P.; Tirrey, J.; Wathey, B. Westman J., Tetradedron2001, 57, 9225-9283.

25. Kalwania, G. S.; Choudhary, S.; Chomal, S. Chemical Science Transactions2014,3(3),
1147-1155.

26. Mathew, V.; Keshavayya,J.; Vaidya, V. P.J Chem. 2007, 4(3),320-342.

27. Singh, G.; Singh, P. A.; Singh, K.; Singh, D. P.; Handa, R. N.; Dubey, S. N. Proc. Nat. Acad. Sci. Ind.2002, 72A,87-94

28. Hassaan, A. M. A. Indian J. Chem.1997, 36,241-243.

29. Soliman, A. A.; Linert ,W. Thermochim. Acta1999, 333,67-75.

30. Nakamoto K. Infrared Spectra of Inorganic and Coordination Compounds, WileyInterscience.NewYork, (1970).

31. Cerchiaro, G.; Saboya, P. L.; Ferreira, A. M. C.; Tomazela, D. M.; Eberlin, M. N. Transit.Met.Chem.2004,29, 495-504.

32. Murukan, B.; Bhageerethi, S. K.; Kochukittan, M.J. Coord. Chem.2007,60,1607-1617.

33. Turan-Zitouni, G.; Kaplancikli, Z. A.; Erol,K.; Kilic, F. S.Farmaco1999,54, 218-223. 